\title{
On the Calculation of SEM and FIB Beam Profiles
}

Jolana Kološová ${ }^{1}$, Tomáš Hrnčír ${ }^{1}$, Jaroslav Jiruše $^{1}$, Miroslav Rudolf ${ }^{1}$ and Jakub Zlámal ${ }^{2,3}$

1. TESCAN Brno, s.r.o., Libušina třída 1, 62300 Brno, Czech Republic

2. Brno University of Technology, Technická 2, 61669 Brno, Czech Republic

${ }^{3 .}$ CEITEC BUT, Technická 10, 61669 Brno, Czech Republic

\section{Introduction and motivation}

Fundamental tasks of charged particle optics, specifically Scanning Electron Microscopy (SEM) and Focused Ion Beams (FIB), include the prediction of the beam profile and the corresponding imaging resolution. For manufacturers of the instruments it is crucial during the development in order to optimize the instrument parameters for given applications. For users it is also important, e.g. for setting the SEM for the best image resolution at the optimum aperture or setting high beam currents at non-optimum aperture. For the scanning electron beam lithography it is necessary to control the exposure pitch, defined as a distance between centers of neighboring spots during the exposure of the resist. Yet another example is the FIB patterning, where the distance of the neighboring spots should match the spot size. Last but not least, for FIB-SEM analytical techniques like Energy Dispersive Spectrometry (EDX), Electron Backscatter Diffraction (EBSD) or Time-of-Flight Secondary Ion Mass Spectrometry (TOFSIMS), it is necessary to control the beam currents and the spot size corresponding to the lateral information limit of given methods.

Most approaches determining the beam profiles suffer from the lack of information about internal optics of microscopes, as their producers usually do not provide complete lens geometry. Thus they compare simulations and simplified theories, as an experimental comparison with a particular instrument is not possible. In this paper we describe the results of calculations, based on detailed manufacturer's knowledge of the instrument optical system. This is compared to experimentally measured resolution and the correlation of these data is discussed.

\section{Review of current methods}

\section{a. Calculation}

Rigorous ways to obtain the exact SEM and FIB beam profiles are direct ray-tracing and wave-optical calculations $[1,2,3]$. The direct ray-tracing can be used in case when the final probe diameter is limited by spherical and/or chromatic aberrations and not by the diffraction. Otherwise the wave-optical calculations must be performed. The wave aberration approximation is usually adopted to calculate the Point Spread Function (PSF) in the image plane (more details are given below), which can be interpreted as the distribution of the beam current [4]. Once the PSF is known, the diameter of the circle containing a certain fraction (e.g. 50\% or $80 \%$ ) of the total probe current can be estimated, as well as the risedistance of the beam swept over a sharp knife-edge, i.e. the edge resolution. Obtained values can be used as parameters defining the electron or ion probe size. It can be shown that $25 \%-75 \%$ edge resolution can be converted to $50 \%$ probe current diameter using $d_{25 \%-75 \%}=0.57 d_{50 \%}$ independently of the particular beam profile [5]. Thus $25 \%-75 \%$ edge resolution has become standard since it roughly corresponds to a radius of the $50 \%$ probe-current spot.

Approximate formulae for the calculation of the geometrical spot diameter are often used when full spot profile calculations are not needed. Such reduction of the full spot profile into a single number is 
sometimes an unacceptable simplification and may lead to wrong estimates. A widely used approximate method is a sum of weighted individual aberration disks together with the demagnified beam source size $[1,4]$. A number of weight coefficients were introduced to compensate for non-Gaussian beams. Our contribution to this topic based on comparison with experimental results will be published elsewhere.

\section{b. Experimental}

From the various methods of resolution measurement [1], the most widely known are the edge resolution (i.e. scanning the beam over a sharp edge and measuring the signal rise-distance) and 2-D Fourier transform analysis of images (i.e. the highest spatial frequency transferred through the microscope defines its resolution limit [6]). For measured edge resolution, various limits of the rise-distance are being used, e.g. $10 \%-90 \%, 25 \%-75 \%$ or $35 \%-65 \%$, see Figure 1 . Therefore, one should always check the definition of rise-distance value. For example, sometimes used $d_{35 \%-65 \%}$ is about a half of the more standard $d_{25 \%-75 \%}$ for the very same edge profile (Figure 1).
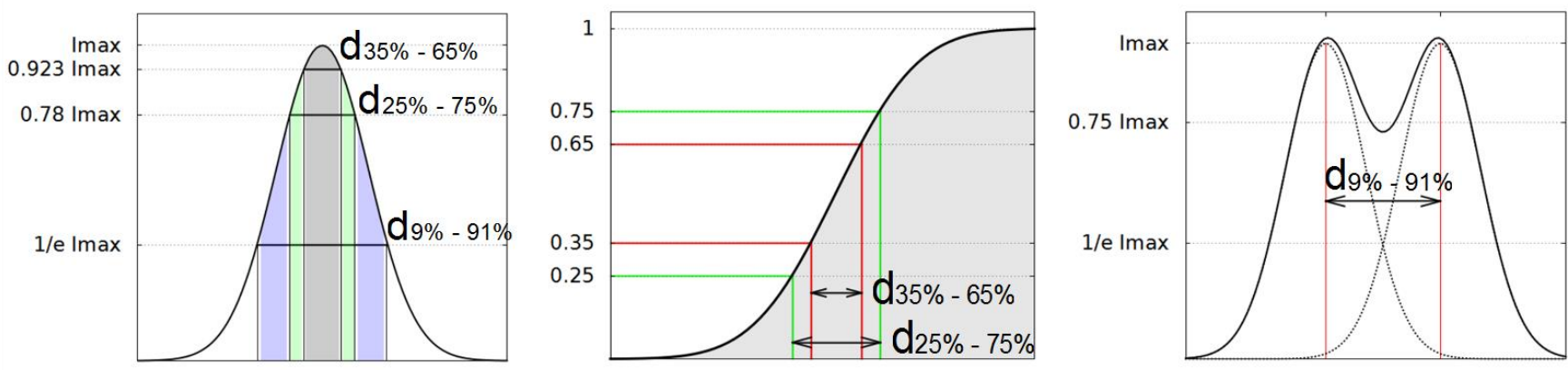

Figure 1. Left: The area of the Gaussian curve shown for several rise-distance limit values of the edge resolution profile. Middle: Different rise-distance limits yield different edge resolution values for the same profile. For example $d_{35 \%-65 \%}=0.57 d_{25 \%-75 \%}$ Right: Two Gaussian spots can be resolved, if they overlap with a separation $d_{9 \%-91 \%} \approx 2 \cdot d_{25 \%-75 \%}$. This is equivalent to the well known Rayleigh criterion of diffraction optics.

\section{Calculation of spot profiles and comparison with experiments}

\section{a. Computation}

We calculated the current density distributions and beam profiles both by direct ray-tracing and by the wave aberration approximation methods. They were incorporated into Electron Optical Design (EOD) [7] software modules. If the resolution is limited by geometric aberrations and not by the diffraction (i.e. the spot diameter broadening caused by the spherical and/or chromatic aberrations is much greater than the Airy disc of the diffraction aberration), the beam profile is calculated by the direct ray tracing.

In case of wave aberration approximation (diffraction cannot be neglected), the monochromatic PSF is calculated $[8,9]$ :

$$
\operatorname{PSF}(r)=\left|\frac{2 \pi}{\lambda} \sqrt{\frac{I_{0}}{\pi \alpha_{\max }}} \int_{\alpha=0}^{\alpha_{\max }} e^{\Phi(\alpha, \Delta z)} J_{0}\left(\frac{2 \pi}{\lambda} r \alpha\right) \alpha d \alpha\right|^{2}
$$

where $I_{0}$ is the beam current, $\alpha_{\max }$ the maximum aperture half angle at the image plane, $\Delta z$ the defocus, $\lambda$ 
the wave length and $J_{0}$ the zeroth order Bessel function of the first kind. Further integration over defocus caused by the chromatic aberration and the beam energy spread gives the polychromatic PSF. Then Line Spread Function (LSF) is calculated:

$$
\operatorname{LSF}(x)=\int_{y=-\infty}^{\infty} \operatorname{PSF}\left(\sqrt{x^{2}+y^{2}}\right) d y, \quad\left(r=x^{2}+y^{2}\right)
$$

and the Edge Spread Function (ESF) is finally obtained as convolution of the LSF and step function (this is equivalent to scanning the calculated spot over a knife edge):

$$
\operatorname{ESF}(x)=\int_{r^{\prime}=-\infty}^{\infty} \chi(r) \operatorname{LSF}\left(r-r^{\prime}\right) d r^{\prime}, \quad \chi(r)=\left\{\begin{array}{l}
1 \text { if } r \geq 0 \\
0 \text { if } r<0
\end{array} .\right.
$$

In both computation methods, accurate models of particular SEM and FIB columns are used in the calculations. Energy spread of the Schottky electron source is taken as $0.7 \mathrm{eV}$ in accordance with the data measured by manufacturer of the emitter [10] and standard operation conditions of the electron guns in TESCAN microscopes.

The calculated resolution is taken as $25 \%-75 \%$ rise-distance of the ESF for several reasons: it is most widely used, it coincides with the beam radius at which the intensity of Gaussian spot drops to 1/e of its maximum value (Figure 1) and a simple conversion between the $d_{25 \%-75 \%}$ and the beam diameter $d_{50 \%}$ is possible, as already mentioned above.

\section{b. Experiments}

The experiments were performed on Schottky FEG SEMs with four different objective lens types (TESCAN MIRA3 and LYRA3 SEM columns). Preliminary ion beam spot profile measurements were performed on FIB column with Gallium Liquid Metal Ion Source (COBRA column of ORSAY PHYSICS).

For SEM, images of gold particles on carbon (standard $\mathrm{Au} / \mathrm{C}$ high resolution test specimen) were acquired at different conditions by varying the beam energy, the working distance and the beam aperture angle.

Automated resolution measurement procedure based on the statistical edge profiling method was used. Edge profiles were extracted from auto-detected edges and statistically processed. The edge resolution was estimated by Gaussian error function fitting to the profiles as $25 \%-75 \%$ raise-distance, see Figures 2 to 5 . Obtained results were then compared with the calculated numbers.

For SEM at low apertures, close to the optimum aperture, the experimental results are in a good agreement with the calculated beam profiles, see Figure 2. If the column setting is far from the optimum aperture, the image resolution becomes much better than the $50 \%$ probe current diameter would suggest, see Figure 3. This is due to the fact that, far from the optimum aperture, the profile of the spot is characterized by a sharp and narrow central peak and wide beam tails, see Figure 4. These tails are reflected in the acquired image only through an increased offset value of the background, which can be 
partially suppressed by optimizing the detector settings. That is why these beam tails do not contribute to the measured image resolution, which is thus given mostly by the width of the central peak.
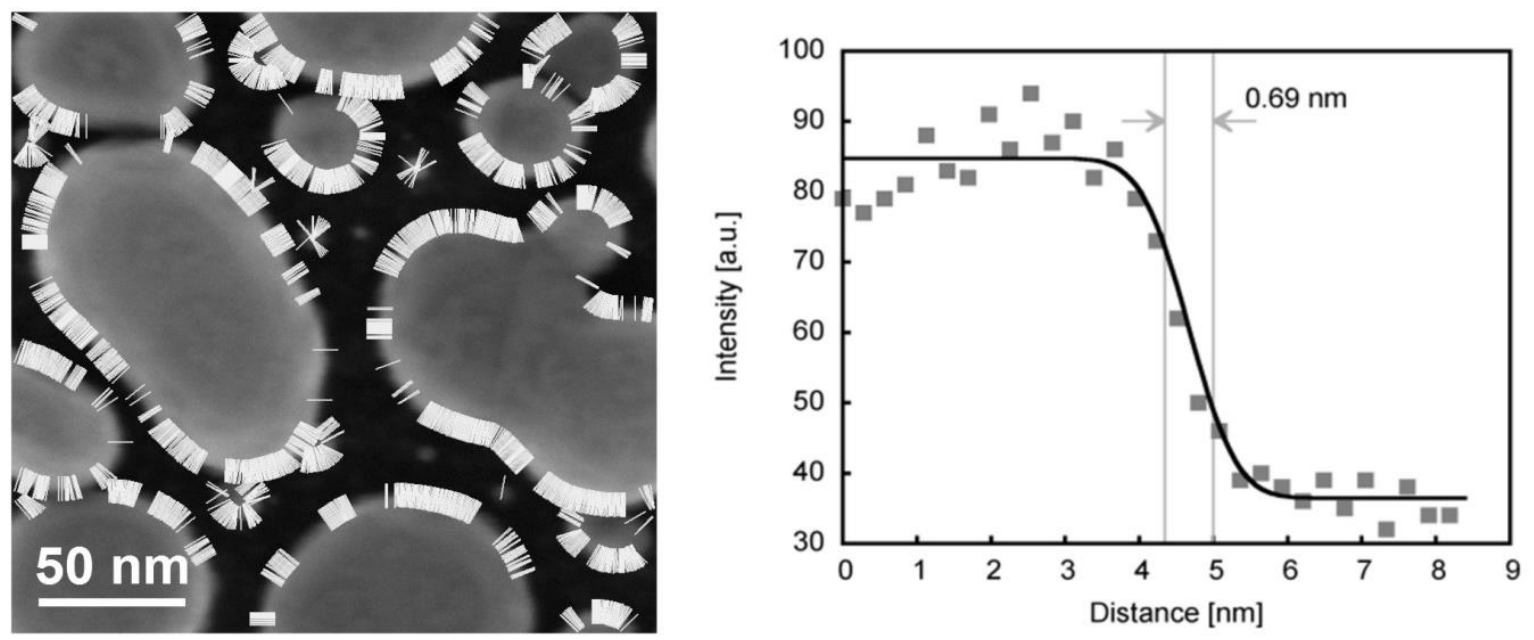

Figure 2. Resolution test of SEM column MIRA3 at the optimum aperture with electron beam energy $30 \mathrm{keV}$. Left: Gold on carbon sample, field of view $217 \mathrm{~nm} .2712$ edge profiles were extracted from automatically detected edges and statistically processed. Right: Gaussian error function fitting to a typical profile with $25 \%-75 \%$ rise-distance of $0.69 \mathrm{~nm}$. This is in agreement with calculated value of $0.62 \mathrm{~nm}$ from ESF function.


Figure 3. SEM resolution test at large final aperture far from optimum at electron beam energy $30 \mathrm{keV}$. Measured imaging resolution is $5.0 \mathrm{~nm}$. The full beam profile was calculated by direct ray tracing, 15 selected energies within the $0.7 \mathrm{eV}$ energy spread were used with 10,000 particles at each energy. Calculated diameter $d_{50 \%}$ of $121 \mathrm{~nm}$ would suggest much worse resolution than actually measured. See the text for discussion. 

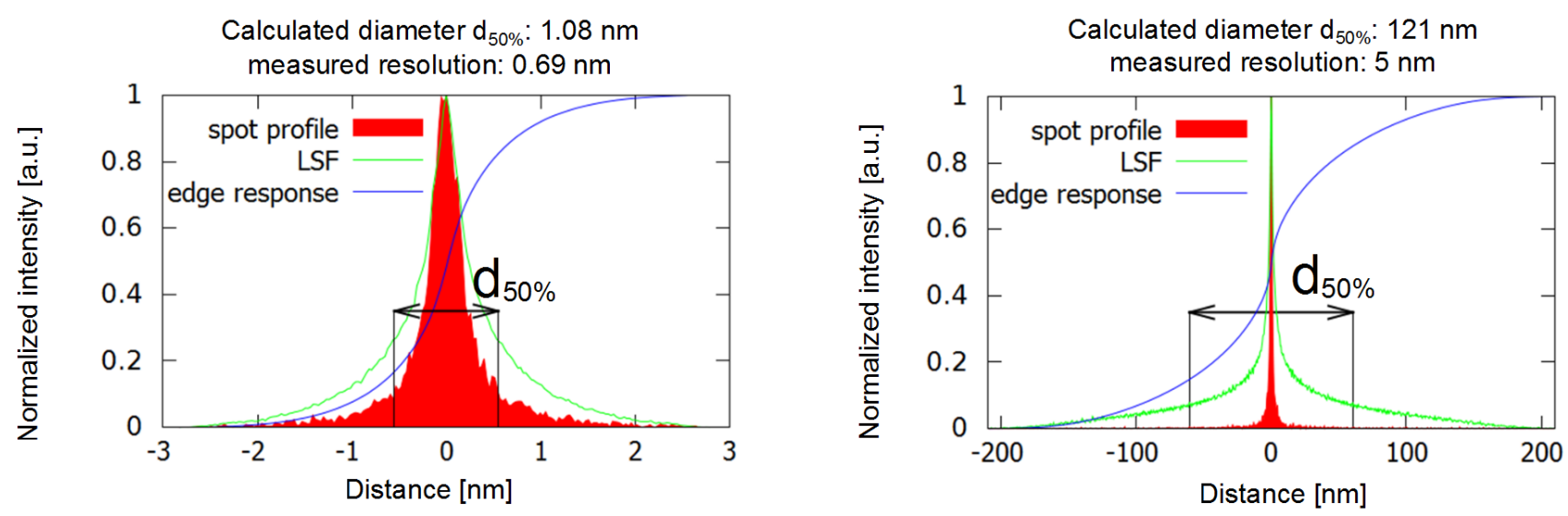

Figure 4. Spot profiles and edge responses calculated for conditions in Figures 2 and 3. Left: Spot profile at small aperture close to optimum - imaging resolution corresponds to the beam diameter $d_{50 \%}$. Right: Spot profile at large aperture far from optimum - imaging resolution corresponds to the central peak of the final spot. Single number $d_{50 \%}$ is no longer sufficient and full beam profile is needed.

This means that the full information about the beam profile is necessary for setting the instrument parameters correctly, especially if the instrument is used at high apertures far from the optimum. Whilst the presence of beam tails is not so important for the imaging, for analytical methods like EDX or EBSD the signal is acquired from the whole spot size and influences the quantitative analysis of results.

Preliminary results of the same procedure in the case of FIB are shown in Figure 5. Further work will be done with wider experimental database including more FIB columns.
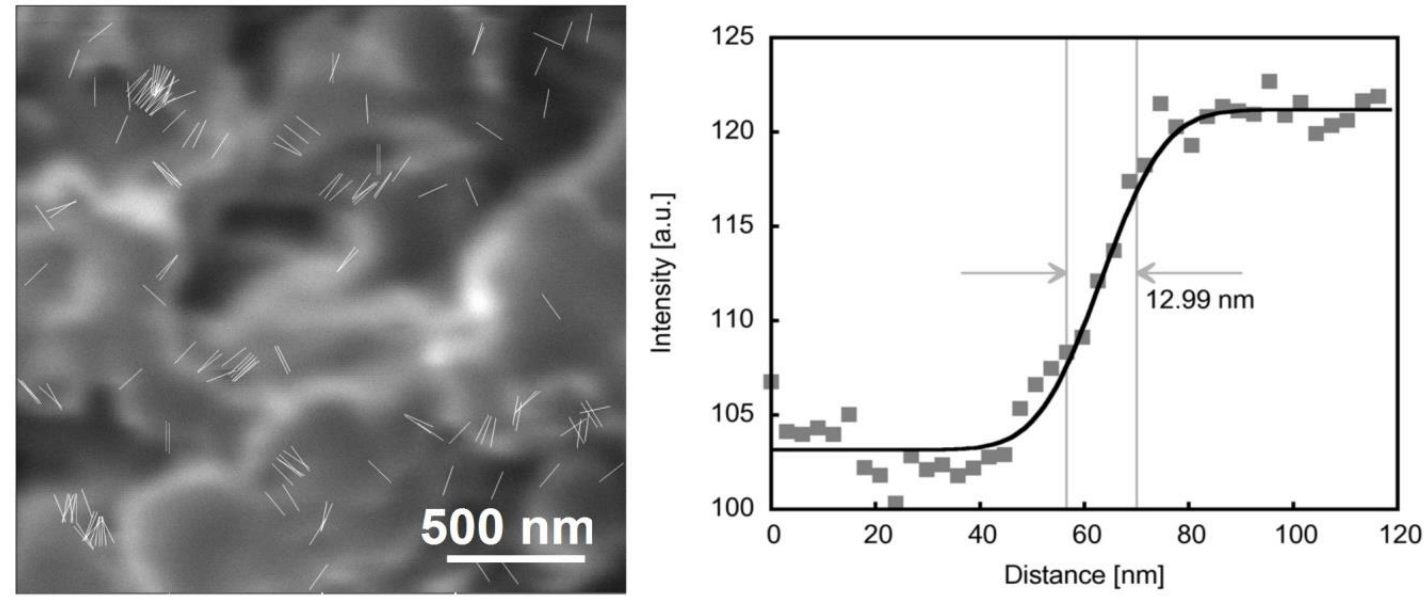

Figure 5. FIB column resolution measurement based on $25 \%-75 \%$ rise-distance (30 keV beam energy, probe current $60 \mathrm{pA}$ ). Measured resolution is $13 \mathrm{~nm}$, resolution calculated from ESF function is $12.9 \mathrm{~nm}$.

\section{Conclusions}

Full spot profile calculations based on wave optical methods give complete information about SEM/FIB spots, and an excellent agreement with the experimental data is obtained for both SEM and FIB columns 
around optimum apertures. At high apertures, far from the optimum, the imaging resolution is far better than simple spot diameter would suggest due to a narrow central peak with a wide background. Therefore, at high apertures the full spot profile calculation is considered the proper way to define beam profiles for practical applications [11].

\section{References:}

[1] L Reimer in "Scanning Electron Microscopy”, (Springer, Berlin), 1998, p. 51.

[2] X Guo et al, Optik 120 (2009), p. 207.

[3] X Zhu et al, SPIE Conf. Proc. Ser. 3777 (1999) p. 35.

[4] M Sato in "Handbook of Charged Particle Optics", 2 nd edition, ed. J Orloff, (CRC Press, Boca Raton) p. 391.

[5] M Bronsgeest, Physics of Schottky Electron Sources, PhD Thesis, TU Delft, 2009.

[6] DC Joy et al, SPIE Proc. Ser. 3998 (2000), p.108.

[7] B Lencova and J Zlamal, Phys. Procedia 1 (2008), p. 315.

[8] E Munro in "Handbook of Charged Particle Optics", $1^{\text {st }}$ edition, ed. J Orloff, (CRC Press LLC, Boca Raton) p. 72.

[9] SW Smith in “The Scientist \& Engineer's Guide to Digital Signal Processing”, (California Technical Publishing), 1997, p.397

[10] S Sakawa et al, Surf. Interface Anal. 35 (2003), p. 11.

[11] The authors acknowledge funding from FR-TI2/736 (MOREMIT) and the European Regional Development Fund (CEITEC-CZ.1.05/1.1.00/02.0068). 